\title{
A-23 \\ COMPORTAMIENTO DE ASTRAGALUS NITIDIFLORUS BAJO DÉFICIT HÍDRICO
}

\author{
Martínez-Sánchez, J.J. Vicente, M.J., Conesa, E., Franco, J.A., Bañón, S.
}

Dpto. de Producción Vegetal. Universidad Politécnica de Cartagena. 30203 Cartagena, España. juan.martinez@upct.es. Proyecto LIFE11 BIO/ES/727 - Conservation of Astragalus nitidiflorus in its potential habitat in the Murcia region

\section{1- Introducción y Objetivos}

Astragalus nitidiflorus («Garbancillo de Tallante») es una leguminosa vivaz endémica del oeste del municipio de Cartagena y que actualmente se encuentra catalogada como "en peligro de extinción». La introducción de ejemplares en el campo es una de las técnicas más empleadas para la conservación de especies en extinción. Se hace por tanto necesario conocer el comportamiento viverístico de $A$. nitidiflorus. Con este trabajo pretendemos conocer la respuesta de la planta al déficit hídrico en términos de crecimiento, desarrollo, estado hídrico e intercambio gaseoso.

\section{2- Materiales y Métodos}

Plántulas de tres meses fueron trasplantadas en abril de 2014 a macetas de PVC de $1,5 \mathrm{~L}(15 \mathrm{~cm} \varnothing)$, que contenían una mezcla de turba rubia y tierra del hábitat (6:1 volumen, respectivamente). Las macetas se dispusieron en un invernadero sobre mesas metálicas, y cada una de ellas tuvo 2 emisores de riego ( $2 \mathrm{I} \mathrm{h}^{-1}$ ).

Cuatros niveles de contenido volumétrico de agua $(\theta)\left(0,40,0,35,0,30\right.$ y $\left.0,25 \mathrm{~cm}^{3} \mathrm{~cm}^{-3}\right)$ fueron mantenidos en el sustrato usando un equipo automático de riego formado por sensores dieléctricos (EC5-Decagon) y un programador-registrador de datos CR1000. Los sensores fueron insertados en el sustrato con su cara más estrecha formando un ángulo de $45^{\circ}$ con la superficie del sustrato, entre los dos emisores y en la cara sur de la maceta. El CR1000 fue programado para regar cuando el $\theta$ del sustrato bajaba del umbral fijado en cada tratamiento, siendo el volumen de un riego el mismo para todos los tratamientos (valor medio aproximado de 290 ml maceta-1 $^{-1}$ mientras que el número de riego lo determinaba el cabezal de riego.

Las temperaturas en el invernadero fueron $15,8 \pm 4,6^{\circ} \mathrm{C}$ (mínima), $34,6 \pm 3,3^{\circ} \mathrm{C}$ (máxima). La humedad relativa $38,83 \pm 12,65 \%$ (mínima) y $78,98 \pm 9,3 \%$ (máxima). La luz diaria integral $12,7 \pm 5,1 \mathrm{~mol} \mathrm{~m}^{-2} \mathrm{~s}^{-1}$

Se midieron el porcentaje de drenaje y la conductividad eléctrica (CE) del drenaje tras cada riego. Al final del experimento se realizaron medidas de crecimiento y desarrollo, se determinó la clorofila SPAD en las hojas, la eficiencia en el uso del agua, el potencial hídrico foliar, la conductancia estomática y la tasa de fotosíntesis (Fig. 1).

Cada tratamiento dispuso sobre una mesas en dos filas de 7 macetas (Fig. 2). Los análisis de regresión fueron realizados con el programa SigmaPlot 10.0 (Systat Software Inc., San Jose, CA).

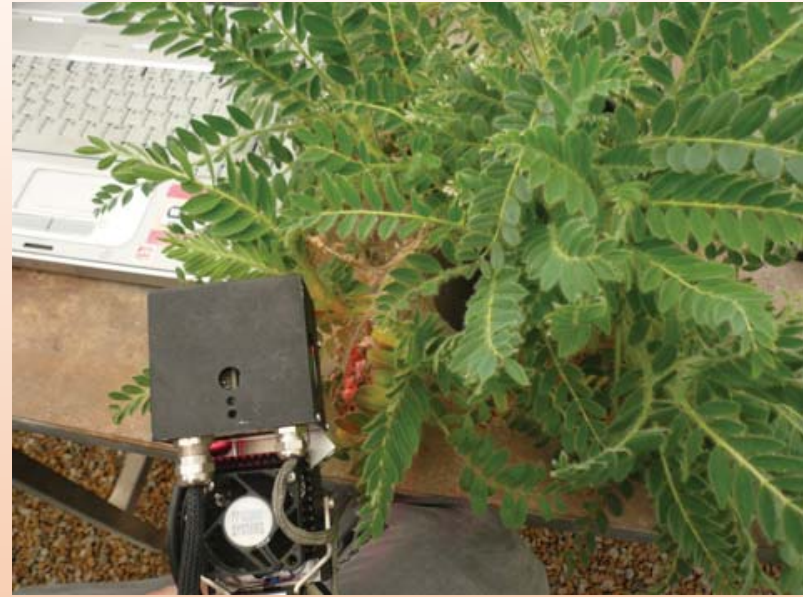

Fig. 1 - Aspecto de A. nitidiflorus

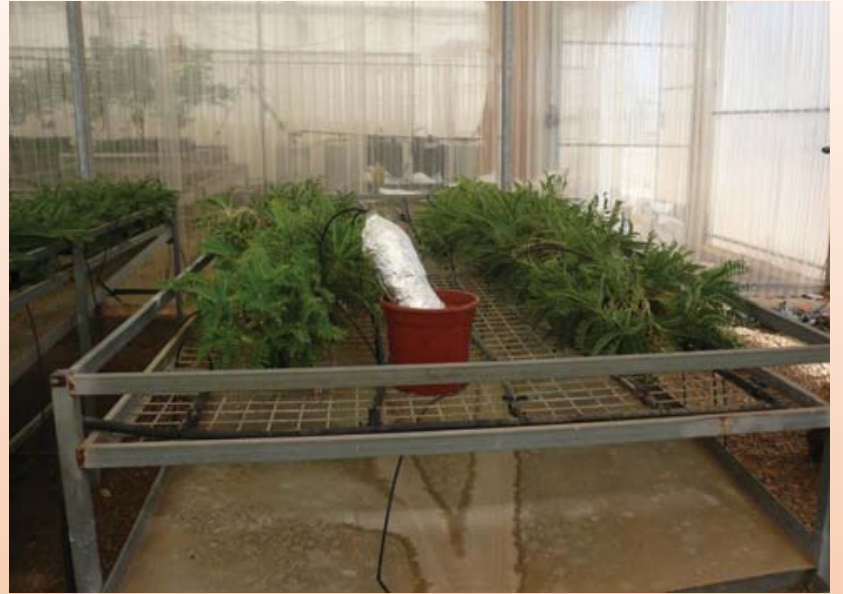

Fig. 2 - Mesa de cultivo

\section{3- Resultados}

Como era de esperar, cuanto más bajo fue el umbral de $\theta$ del sustrato menor fue el número de riegos (Tabla 1 ). Esto fue muy evidente entre $0,40,0,35$ y $0,30 \mathrm{~cm}^{3}$ $\mathrm{cm}^{-3}$, mientras que entre los tratamientos de 0,30 y 0,25 sólo hubo una diferencia de tres riegos. Como el volumen de riego fue el mismo para todos los tratamientos, el agua aplicada por maceta siguió un comportamiento similar al número de riegos. Así, las macetas bajo el umbral de $0,40 \mathrm{~cm}^{3} \mathrm{~cm}^{-3}$ recibieron aproximadamente el doble de agua $\left(31,2\right.$ I maceta $\left.^{-1}\right)$ que las plantas bajo los tratamientos de 0,30 y 0,25 , y estos dos últimos tratamientos recibieron una cantidad de agua similar. Conforme se reducía el umbral de $\theta$ que decidía el riego el drenaje tendía a aumentar, mientras que la CE del drenaje tuvo una respuesta contraria.

Se obtuvieron relaciones lineales significativas entre el $\theta$ del sustrato y la altura de planta, anchura de planta, peso seco aéreo, área foliar, área del limbo y número de hojas (Figs. 3A, B, C, D y E). La altura fue menos sensible a la disminución del $\theta$ que la anchura (Fig. 3A). El peso seco aéreo y el área foliar estuvieron fuertemente relacionado con el $\theta$, descendiendo con la bajada del $\theta$ en el sustrato. La disminución del $\theta$ redujo la eficiencia en el uso del agua consumida expresada como la relación entre el peso seco y el consumo de agua (Fig. 3F).

El contenido relativo de clorofila foliar (SPAD) disminuyó conforme el $\theta$ descendía (Fig. 4A), pero este descenso fue pequeño. La fotosíntesis, conductancia estomática y potencial hídrico foliar medidos al mediodía al final del experimento no fueron afectados por los tratamientos (Figs. 4B, C y D).

\section{4- Discusión}

La disminución de la humedad en el sustrato redujo fuertemente al crecimiento foliar de $A$. nitidiflorus, lo que fue evidente por debajo de un $\theta$ en el sustrato de 0,35 $\mathrm{cm}^{3} \mathrm{~cm}^{-3}$. Esto explica el aumento de la EUA de esta planta conforme aumenta la humedad en el sustrato. Sin embargo, bajos niveles de humedad en el sustrato más bajos no produjeron daños foliares en forma de necrosis. E incluso no hubo clorosis foliares, a pesar de la caída de la clorofila SPAD en las hojas con la reducción del $\theta$ del sustrato, porque visualmente no se observaron diferencias en el verdor de las hojas.

La respuesta contraria del porcentaje y la CE del drenaje a la humedad del sustrato puede estar relacionada con la capacidad de retención de agua del sustrato, puesto que un sustrato que se mantiene bien humedecido a lo largo del cultivo (uniformemente y constantemente humedecido), como ocurrió aquí con los dos valores de $\theta$ mayores, tiene mejor aptitud para retener agua que un sustrato mal humedecido porque evita problemas de rehidratación y canalización del agua (Valdés et al., 2014). Problemas de rehidratación y de canalización pudieron contribuir a que el lavado de sales en las macetas bajo 0,25 y $0,30 \mathrm{~cm}^{3} \mathrm{~cm}^{-3}$ fuera poco eficaz, según los datos de la CE de los drenajes. 

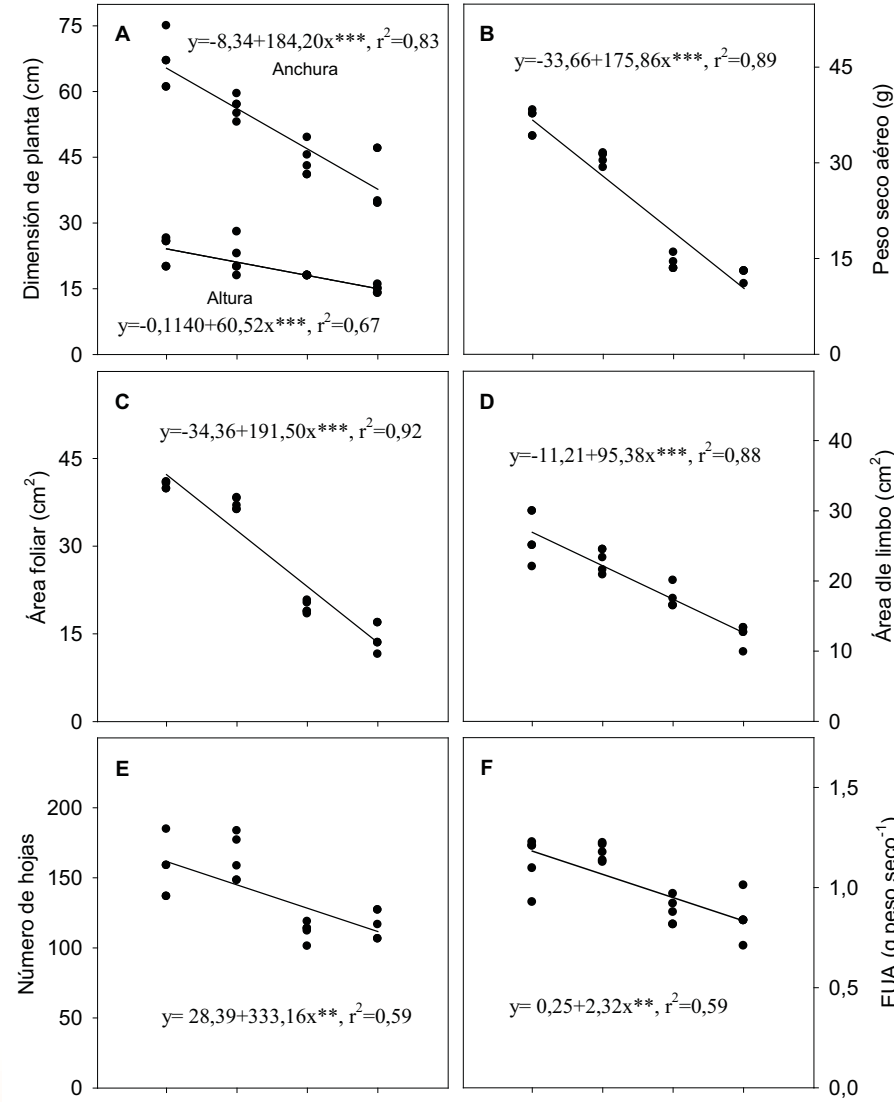

$0,40 \quad 0,35 \quad 0,30 \quad 0,25$
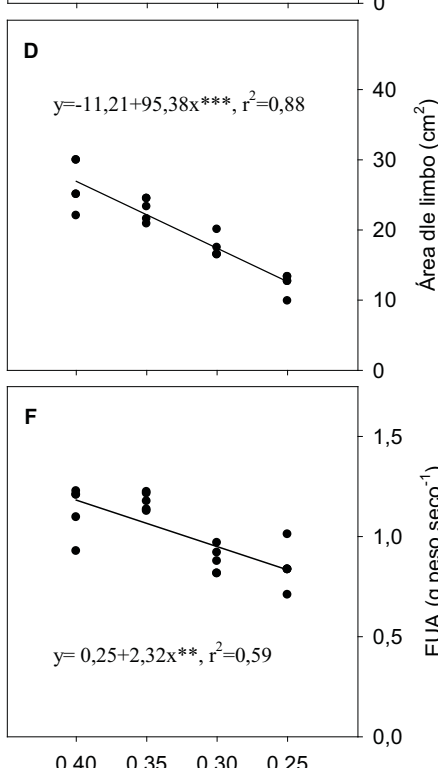

Contenido volumétrico de agua en el sustrato $\left(\mathrm{cm}^{3} \mathrm{~cm}^{-3}\right)$

Fig. 3 - Parámetros de crecimiento y desarrollo. **,*** indican significación estadística con $P<0,05$ y $P<0,001$, respectivamente.

\begin{tabular}{lcccc} 
Tabla 1 - Parámetros de riego & \multicolumn{5}{l}{} \\
\hline \multirow{2}{*}{ Parámetros } & \multicolumn{4}{c}{ Contenido volumétrico de agua $\left(\mathrm{cm}^{3} \mathrm{~cm}^{-3}\right)$} \\
\cline { 2 - 5 } & 0,40 & 0,35 & 0,30 & 0,25 \\
\hline Número de riegos & 104 & 86 & 55 & 52 \\
Agua aplicada (litros maceta $\left.{ }^{-1}\right)$ & 31,2 & 25,8 & 16,5 & 15,6 \\
Porcentaje de drenaje & 7,78 & 9,85 & 17,54 & 16,87 \\
CE del drenaje $\left(\mathrm{dS} \mathrm{m}^{-1}\right)$ & 2,61 & 2,05 & 1,71 & 1,59 \\
\hline
\end{tabular}
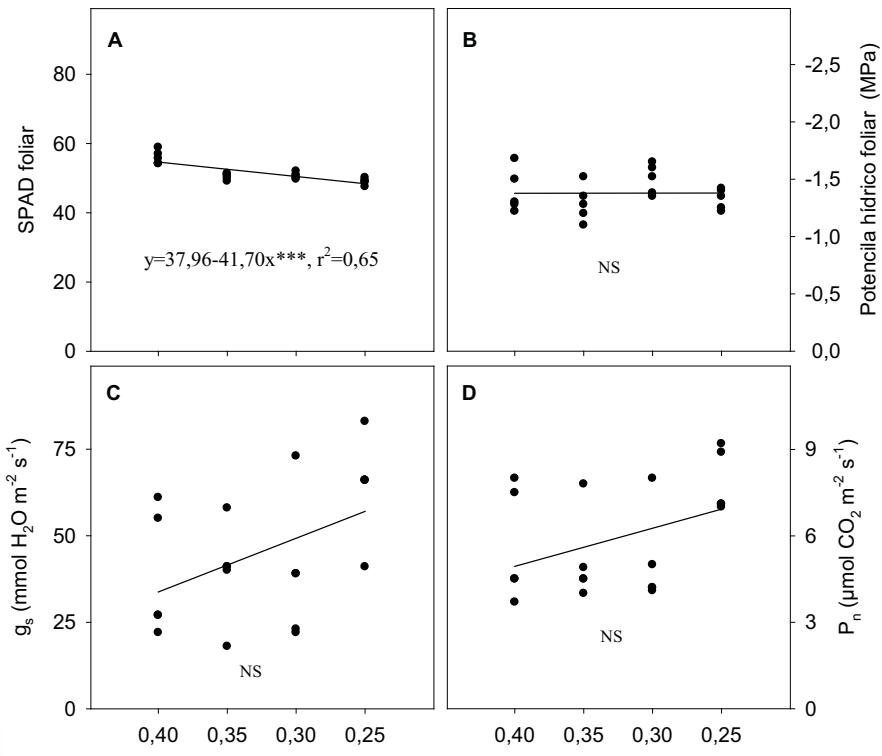

Contenido volumétrico de agua en el sustrato $\left(\mathrm{cm}^{3} \mathrm{~cm}^{-3}\right)$

Fig. 4 - Parámetros de intercambio gaseoso y SPAD. NS, ${ }^{* * *}$ indican ausencia de significación estadística o significación estadística con $P<0,001$ respectivamente.

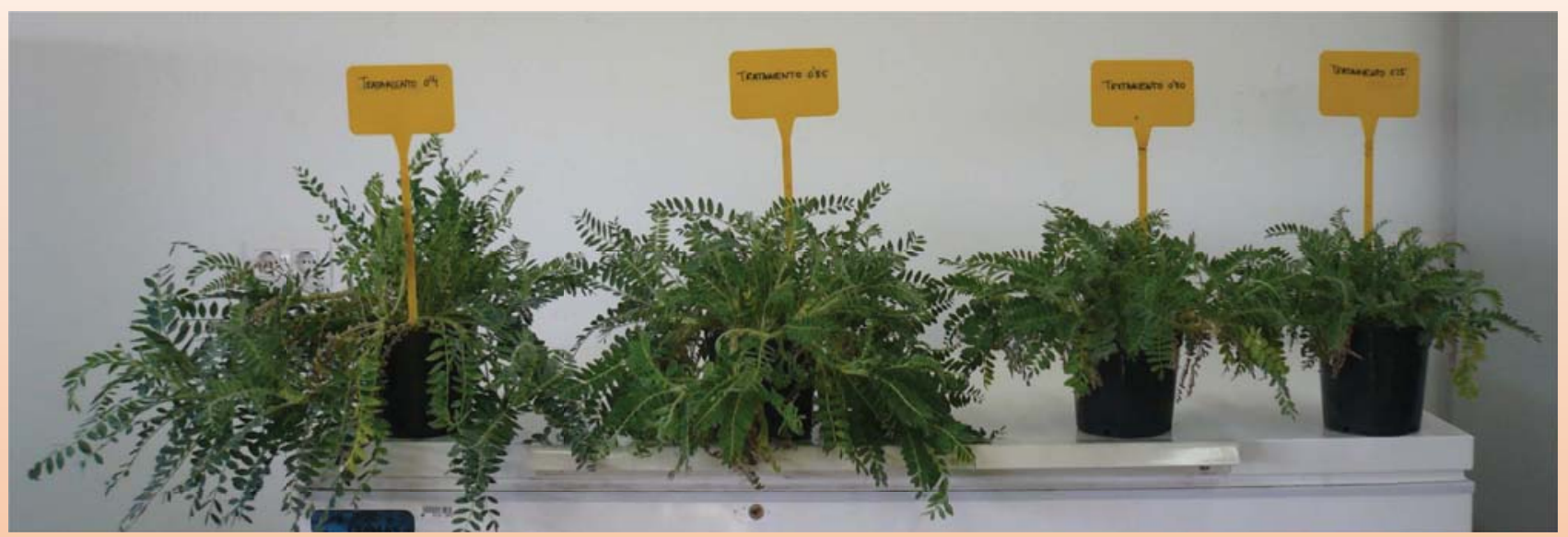

Fig. 5 - Aspecto de las plantas al final del experimento según tratamientos

Es bien conocido que las plantas pueden adaptarse a condiciones de sequía mediante cambios morfológicos y fisiológicos. En este experimento fueron evidentes los primeros (reducción del crecimiento aéreo por déficit hídrico, Fig. 5), lo que sugiere que $A$. nitidiflorus reduce su superficie foliar y/o de su biomasa seca como mecanismos de evitación frente a la sequía. Una disminución del crecimiento foliar suele conducir a la reducción de fotoasimilados, y por tanto a la caída de la producción de biomasa seca de la planta, como aquí sucedió. El crecimiento de $A$. nitidiflorus fue más sensible al déficit hídrico que el intercambio gaseoso, lo que justificaría la caída de la EUA con la bajada del $\theta$ en el sustrato. Sin embargo, $A$. nitidiflorus no mostró cambios adaptativos por déficit hídrico promovidos a través de mecanismos fisiológicos relacionados con la $P_{\mathrm{n}}$ y $g_{\mathrm{s}}$. Quizás, un seguimiento a lo largo del ensayo del intercambio gaseoso hubiera mostrado diferencias de $P_{\mathrm{n}}$ y $g_{\mathrm{s}}$ entre tratamientos, e incluso en un estudio evolutivo a lo largo del día,

\section{5- Conclusiones y recomendaciones}

- Astragalus nitidiflorus responde al déficit hídrico reduciendo su tamaño y biomasa seca aérea, especialmente reduciendo el crecimiento foliar

- Esta especie exhibió una buena regulación estomática bajo condiciones de luz y calor

- A. nitidiflorus se adapta bien al déficit hídrico sin presentar daños foliares ni clorosis foliares

- La reducción de la humedad en el sustrato disminuye la eficiencia en el uso del agua en $A$. nitidiflorus

- Recomendamos regar esta planta manteniendo bajos niveles de humedad en el sustrato, porque mejora su compacidad sin sufrir fisiológicamente, y además se reduce el consumo de agua

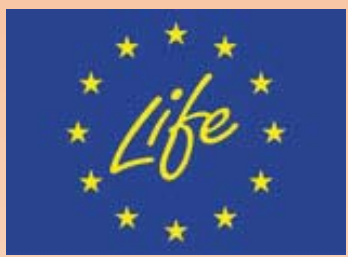

